\title{
Production of low mass electron pairs due to the photon-photon mechanism in central collisions
}

\author{
Kai Hencken and Dirk Trautmann \\ Institut für theoretische Physik der Universität Basel, Klingelbergstrasse 82, CH-4056 Basel, Switzerland \\ Gerhard Baur \\ Institut für Kernphysik (Theorie), Forschungszentrum Jülich, D-52425 Jülich, Germany
}

(Received 25 August 1999; published 19 January 2000)

\begin{abstract}
We calculate the probability for dilepton production in central relativistic heavy ion collisions due to the $\gamma \gamma$ mechanism. This is a potential background to more interesting mechanisms. We find that this mechanism is negligible in the CERES experiments. Generally, the contribution due to this mechanism is small in the central region, while it can be large for small invariant masses and forward or backward rapidities. A simple formula based on the equivalent photon approximation and applications to a possible scenario at the Relativistic Heavy Ion Collider are also given.
\end{abstract}

PACS number(s): 25.75.- q, 12.20.-m, 34.50.- $\mathrm{s}$

In peripheral relativistic heavy ion collisions there are huge effects due to the strong electromagnetic fields, while strong interactions among the colliding ions can be virtually neglected, due to their short range [1,2]. In central collisions, on the other hand, the strong interactions between the colliding ions completely overwhelm the effects due to the electromagnetic interaction. It is the purpose of this paper to study the effects due to the interactions of the very strong (coherent) electromagnetic fields in the central collisions, see Fig. 1. The modification of the fields during the collision due to the strong interactions will be neglected for simplicity. We are mainly interested in the order of magnitude of the effects. Not unexpectedly, the production of strongly interacting particles (like $\pi_{0}$ ) via the $\gamma \gamma$ mechanism is negligible. On the other hand, the photon-photon mechanism could play a role in the production of $e^{+} e^{-}$pairs (and also muon pairs). In recent experiments [3] low mass electron pairs are measured in $p$ - $\mathrm{Be}, p-\mathrm{Au}$, and $\mathrm{S}-\mathrm{Au}$ collisions. Even heavier systems like $\mathrm{Au}-\mathrm{Pb}$ have recently been studied [4]. For the proton induced interactions, the spectra are explained essentially by electron pairs from hadronic decays, whereas for the heavier systems an enhancement is obtained, which is suggested to come from two-pion annihilation. In this paper we are going to calculate the contribution of the $\gamma \gamma$ mechanism to the electron pairs.

First the general theoretical framework is presented. Calculations within the framework of lowest order QED [5] are then given and compared to order of magnitude estimates using the equivalent photon approximation. In these calculations we have used the same kinematical constraints as are used in the CERES experiment. We also present calculations for a possible scenario at the Relativistic Heavy Ion Collider (RHIC).

The vector potential of the combined electromagnetic field of two heavy ions, which travel with constant four velocities $u^{(1,2)}$ and with an impact parameter $b$ between them is given by

$$
\begin{aligned}
A_{\mu}(q)= & -2 \pi e \frac{1}{q^{2}}\left[Z_{1} F_{1}\left(q^{2}\right) u_{\mu}^{(1)} \delta\left(q u^{(1)}\right) \exp (i q b / 2)\right. \\
& \left.+Z_{2} F_{2}\left(q^{2}\right) u_{\mu}^{(2)} \delta\left(q u^{(2)}\right) \exp (-i q b / 2)\right]
\end{aligned}
$$

where the four-velocities are given by $u^{(1)}=(\gamma, 0,0, \gamma \beta)$ and $u^{(2)}=(\gamma, 0,0,-\gamma \beta)$ [6]. In the following we restrict ourselves to central collisions with $b \approx 0$. The nuclear charges and form factors are denoted by $Z_{i}$ and $F_{i}\left(q^{2}\right)$, respectively $(i=1,2)$. It should be noted that due to the strong interaction between the heavy ions, the nuclear charge distributions (which give rise to the electromagnetic field) will change; both stopping, as well as a spreading out, will take place. Such effects were considered in [7]. In addition there is also pair production from the stopping itself [8]. This pair production due to the bremsstrahlung will strongly depend on the actual stopping. We will neglect these stopping effects here. The collision of the two electromagnetic fields (equivalent photons) can then give rise to all kind of final states $f$, like $e^{+} e^{-}$pairs, $\pi_{0}$, etc.

The matrix element for $e^{+} e^{-}$pair production is given by

$$
\begin{aligned}
M= & -i e^{2} \bar{u}\left(p_{-}\right) \int \frac{d^{4} p}{(2 \pi)^{4}} A\left(p_{-}-p\right) \\
& \times \frac{\not p+m}{p^{2}-m^{2}} A\left(p_{+}+p\right) \vee\left(p_{+}\right),
\end{aligned}
$$

where $\bar{u}\left(p_{-}\right)$and $\mathrm{v}\left(p_{+}\right)$are the Dirac spinors describing the produced electron and positron, respectively. For impact pa-

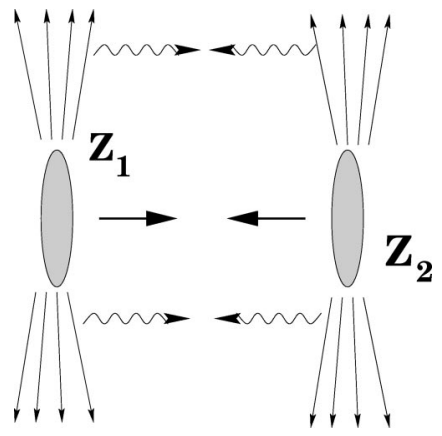

FIG. 1. The strong electromagnetic fields surrounding the heavy ions are also present in central collisions $(b=0)$ and will lead to pair production processes due to the $\gamma \gamma$ fusion mechanism. 
rameter $b$ equal to zero, this can be calculated completely analytically as was done in [5]. The integration over all final states was then done with a MC integration $[9,10]$ in the program code BORNZERO. The form factor of a pointlike charge distribution $[F(q)=1]$ contains unrealistically high Fourier components, which can lead to spurious effects. Already the total probability diverges logarithmically with $b$ $\rightarrow 0$ in this case, see [6]. In order to get realistic results a nuclear form factor is needed in the calculations. We use here either a monopole form factor

$$
F_{\text {monopole }}\left(q^{2}\right)=\frac{\Lambda^{2}}{\Lambda^{2}-q^{2}},
$$

where $\Lambda$ is chosen to reproduce the correct rms radius of the nucleus, as well as, one which is the sum of two monopole form factors. We use them as a check on the sensitivity of our results on the detailed form of the form factor. Compared to a "realistic" form factor, the monopole form factor does not fall off rapidly enough for large $q$. On the other hand, this means that our result is an upper bound.

The impact parameter dependent equivalent photon number $N(\omega, \rho)$ for a monopole form factor is given as $[11,5]$

$$
\begin{aligned}
N(\omega, \rho)= & \frac{Z^{2} \alpha}{\pi^{2}} \mid \frac{\omega}{\gamma} K_{1}\left(\frac{\omega}{\gamma} \rho\right)-\left[\frac{\omega^{2}}{\gamma^{2}}+\Lambda^{2}\right]^{1 / 2} \\
& \times\left. K_{1}\left(\left[\frac{\omega^{2}}{\gamma^{2}}+\Lambda^{2}\right]^{1 / 2} \rho\right)\right|^{2} .
\end{aligned}
$$

A simple approximation neglecting the contribution from the nuclear interior is

$$
N(\omega, \rho)=\frac{Z^{2} \alpha}{\pi^{2} \rho^{2}}, \quad R<\rho<\gamma / \omega
$$

and 0 otherwise. The radius of the ion is given by $R$ $=1.2 \mathrm{fm} A^{1 / 3}$. With this expression a simple formula for the product of the equivalent photon numbers integrated over $\rho$ can be given:

$$
\int d^{2} \rho N\left(\omega_{1}, \rho\right) N\left(\omega_{2}, \rho\right) \approx \frac{Z_{1}^{2} Z_{2}^{2} \alpha^{2}}{\pi^{3} R^{2}}\left[1-a^{2} e^{2|Y|}\right],
$$

where $Y=1 / 2 \ln \left(\omega_{1} / \omega_{2}\right)$ and $a=R_{>} M / 2 \gamma<1$, with $R_{>}$the larger one of the two nuclear radii and $M$ the invariant mass of the produced system.

In the EPA the transverse momentum of electron and positron are the same but in opposite directions $\left[\vec{p}_{t}\left(e^{+}\right)=\right.$ $\left.-\vec{p}_{t}\left(e^{-}\right)\right]$. We have the following expression for the probability to emit an $e^{+} e^{-}$pair with rapidities $y_{+}$and $y_{-}$and transverse momentum $p_{t}\left(e^{+}\right)$

$$
\frac{d^{4} P}{d^{2} p_{t} d y_{+} d y_{-}}=\int d^{2} \rho N\left(\omega_{1}, \rho\right) N\left(\omega_{2}, \rho\right) \frac{1}{\pi} \frac{d \sigma}{d \hat{t}},
$$

where $d \sigma / d \hat{t}$ is the cross section for the subprocess $\gamma+\gamma$ $\rightarrow e^{+}+e^{-}$, see, e.g., [12].

Similarly the probability to produce a final state with invariant mass $M$ and rapidity $Y$ is given by

$$
\frac{d^{2} P}{d M d Y}=\frac{Z_{1}^{2} Z_{2}^{2} \alpha^{2}}{\pi^{3} R^{2}}\left[1-a^{2} e^{2|Y|}\right] \frac{2 \sigma_{T T}\left(m^{2}\right)}{m},
$$

where $\sigma_{T T}$ is the corresponding $\gamma+\gamma \rightarrow f$ photon-photon fusion cross section.

From this equation it can be easily seen that, e.g., $\pi_{0}$ production is very small:

$$
\frac{d P}{d y}=\frac{8 Z_{1}^{2} Z_{2}^{2} \alpha^{2} \Gamma_{\gamma \gamma}\left(\pi^{0}\right)}{\pi R^{2} M_{\pi}^{3}}\left[1-a^{2} e^{2|Y|}\right] .
$$

For $Y=0$ we find (for $\gamma$ large enough so that $a \ll 1$ ) that the pion production probability $d P / d Y \approx 1.3 \times 10^{-5}$ for $\mathrm{Pb}-\mathrm{Au}$ or $\mathrm{Au}-\mathrm{Au}$ collisions.

Since the $\pi^{0}$ is the lightest hadron, it is also the hadronic state which will be produced with the highest probability. Therefore the production of hadronic final states by the electromagnetic fields will be negligible compared to the hadronic production. We can therefore concentrate in the following only on $e^{+} e^{-}$pairs, similar calculations are of course also possible for muon or $\tau$ pairs. As there was some concern regarding $e^{+} e^{-}$pairs coming from the electromagnetic production in connection with the experiments [13], we calculate them here exactly.

We want to compare our results directly with the measurements of the CERES experiment $[3,4]$ and also want to make a prediction for a possible scenario at RHIC [14]. For comparison with CERES we have used the same kinematical restriction $\left(p_{t}>200 \mathrm{MeV}\right.$ for both $\mathrm{S}-\mathrm{Au}$ and $\mathrm{Pb}-\mathrm{Au}$ collisions). The CERES experiment also has a restriction on the relative angles between electron and positron $\left(\Theta_{e e}\right.$ $>35 \mathrm{mrad})$. This was not taken into account, as tests have shown that - apart from the very small invariant masses this condition was always fulfilled.

The results of our calculations are shown in Figs. 2-4. We compare directly $\left(d^{2} N_{e e} / d y d m_{e e}\right) /\left(d N_{c h} / d y\right)$, using $d N_{c h} / d y=125$ for S-Au collisions, $d N_{c h} / d y=235$ (the average of two experimental runs of CERES for $\mathrm{Pb}-\mathrm{Au}$ collisions.) We show the quantity $d^{2} N / d y d m_{e e}$, which is defined to be the differential probability with respect to the $y$ of either the electron or the positron and integrated over the allowed $y$ range of the other $(2.1<y<2.65$ in the lab frame, corresponding to $-0.93<y<-0.38$ in the "center of velocity" frame), following [3].

We note the following points: The results of our calculations are well below the experimental results of [3] and [4]. Since the cross-section for the $\gamma \gamma$ mechanism scales with $Z_{1}^{2} Z_{2}^{2}$ we expect the strongest effects for the heaviest systems. For the $p$ - $\mathrm{Be}$ and $p$-Au case at $450 \mathrm{GeV}$ [3], the $\gamma \gamma$ mechanism is only a small fraction, as expected. We do not need to show this. Even for the heaviest systems [4], the effects are well below the measurements. Therefore we can 


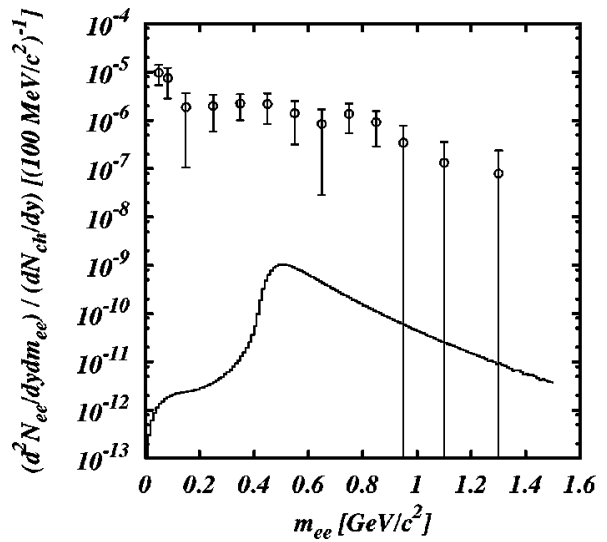

FIG. 2. $d^{2} N_{e e} / d y d m_{e e}$ is shown for the electromagnetic electron-positron pair production for central S-Au collisions at CERES $\left(p_{t, \min }>200 \mathrm{MeV} / c\right)$; also shown are the experimental results of the CERES experiment [3].

safely conclude that the $\gamma \gamma$-mechanism plays only an insignificant role for the experimental results of $[3,4]$.

For the RHIC scenario, see Fig. 4, we have used the experimental conditions as discussed in [14]: $p_{t}>200 \mathrm{MeV}$, $-0.35<y<0.35 . d N_{c h} / d y$ was assumed to be 1100 . We compare our results with the theoretical predictions of [14] for central collisions. In these calculations in-medium modifications of the $\omega$ and $\rho$ meson were taken into account. Again the $\gamma \gamma$ mechanism is only a small background compared to the pairs coming from hadron decay.

Calculations with both the single and the double monopole form factor are shown for $\mathrm{Pb}-\mathrm{Au}$ and $\mathrm{Au}-\mathrm{Au}$ collisions. The results for the CERES experiment are found to be sensitive to the form of the factor both at low and high invariant masses, those for RHIC only for the low-invariant mass region $\left(m_{e e}<2 p_{t}\right)$. The sensitivity in this range is rather easy to understand: In order to be in a low invariant mass state, even though the momenta is at least $p_{t}$, electron and positron cannot be produced in opposite transverse directions, as is assumed in the equivalent photon approximation. In the EPA the invariant mass of the lepton pair is given by

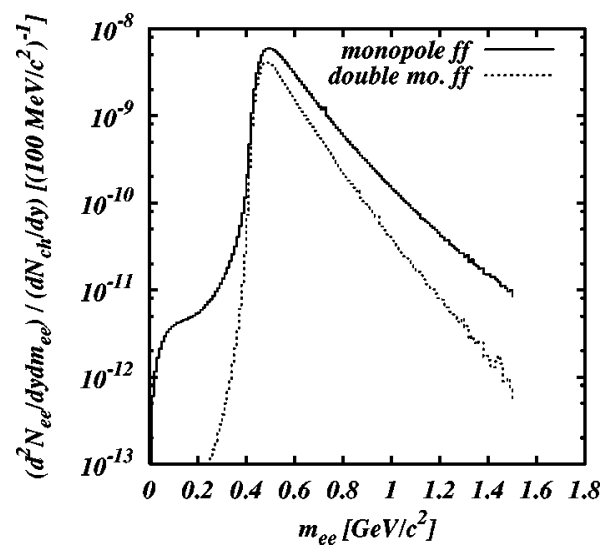

FIG. 3. $d^{2} N_{e e} / d y d m_{e e}$ is shown for the electromagnetic electron-positron pair production for central $\mathrm{Pb}-\mathrm{Au}$ collisions at CERES $\left(p_{t, \min }>200 \mathrm{MeV} / c\right)$ and for two different form factors; see text for details.

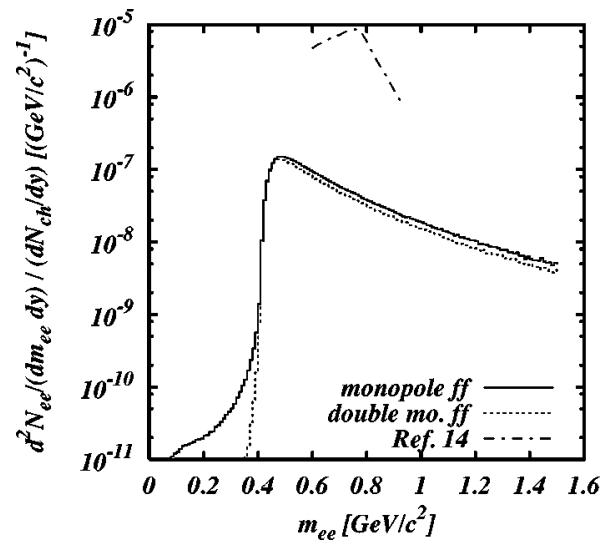

FIG. 4. Same as Fig. 3 for central Au-Au collisions at RHIC. Also shown as dashed-dotted line is the theoretical calculation, given in [14], coming from the hadronic interactions. See also text for details.

$$
m_{e e}=2 p_{\perp} \cosh \left[1 / 2\left(y_{+}-y_{-}\right)\right] .
$$

Therefore the sum of their momenta is larger than the typical range of the form factor (about $50 \mathrm{MeV}$ for $\mathrm{Pb}$ or $\mathrm{Au}$ ). This makes them sensitive to the high $q$ part of the form factor.

The discrepancy at higher invariant masses at the CERES experiment comes from a different reasoning: In order to produce a pair with, for example, $m_{e e}=1 \mathrm{GeV}$ and with $Y$ $=0$, one needs photons with energy about $\omega=500 \mathrm{MeV}$ (estimated from the equivalent photon approximation). The kinematics of this process relates this with the virtuality of the photon:

$$
Q^{2}=q_{\perp}^{2}+(\omega / \gamma)^{2} .
$$

With $\gamma \approx 10$ the virtuality $Q^{2}$ of the photon is then at least 50 $\mathrm{MeV}$ at CERES, but only at least $7 \mathrm{MeV}$ at RHIC. Therefore at CERES one is again sensitive to the form factor at large virtuality.

For an order of magnitude estimate, we can use the EPA. We use Eq. (7) together with the equivalent photon number $N$ of Eq. (4) and the exact expression for $d \sigma / d \hat{t}$, see, e.g., [15], and integrate over $d^{2} p_{t}$.

One can find also a simple analytic formula for the probability to produce dileptons in the central region. We can neglect the square of the electron mass as compared to the values of the Mandelstam variables $\hat{s}$ and $\hat{t}$ of the subprocess $\gamma+\gamma \rightarrow e^{+}+e^{-}$and find

$$
\frac{d \sigma}{d \hat{t}}=\frac{2 \pi \alpha^{2}}{\hat{s}^{2}}\left(\frac{\hat{s}+\hat{t}}{\hat{t}}+\frac{\hat{t}}{\hat{s}+\hat{t}}\right) .
$$

For $y_{+} \approx y_{-}$we have $\hat{t}=-\hat{s} / 2$ and we find

$$
\frac{d N_{e e}}{d m_{e e}} \approx(\Delta y)^{2} 2 \frac{Z_{1}^{2} Z_{2}^{2} \alpha^{4}}{\pi^{2} R^{2} m^{3}} .
$$

In Fig. 5 the different approaches are compared both for $\mathrm{Au}-\mathrm{Au}$ collisions at RHIC $(-0.35<y<0.35, \Delta y=0.7)$ and 


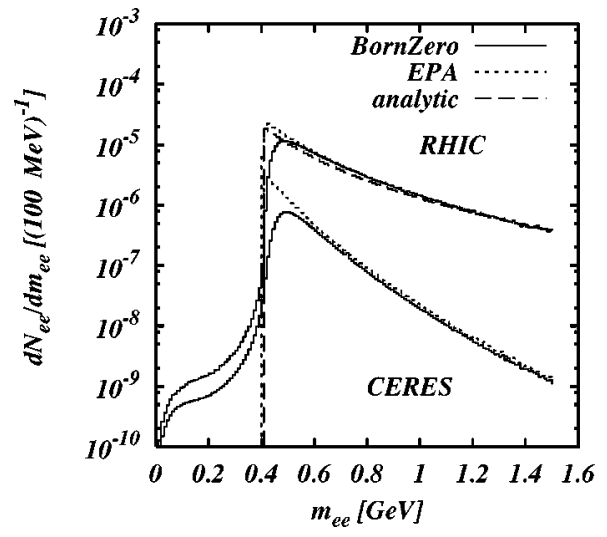

FIG. 5. Different approximations to the pair production probability are compared. Shown are the results of the full calculation (BornZero), the full EPA calculation [EPA, Eq. (7) with $N$ of Eq. (4)] and the analytic expression of Eq. (13) (analytic).

$\mathrm{Pb}$-Au collisions at CERES $(2.1<y<2.65, \Delta y=0.55)$. One finds that EPA agrees quite well at invariant masses above $2 p_{t}$ with the exact calculation. The analytic expression given above is in good agreement with the results at RHIC, but as explained above, is not valid for the conditions at CERES, as the parameter $a$ is larger than 1 [EPA would predict in this case $(a>1)$ a value of zero]. Equation (12) can be rewritten in terms of a differential cross section $d \sigma / d \Omega$, which results in a $\left[1+\cos ^{2}(\theta)\right] / \sin ^{2}(\theta)$ dependence on the scattering angle in the center of mass system. From this one sees that the importance of the $\gamma \gamma$ mechanism, when one goes to more forward or backward angles (larger rapidities), increases as compared to the other mechanisms for dilepton production, see also [7].

A comparison with the measured $e^{+} e^{-}$pairs in central collisions at CERES shows, that pairs produced electromagnetically from the strong electric fields, are only a small background. We think that this calculation has, for the first time, convincingly shown that the $\gamma \gamma$ pairs are negligible. This is mainly due to the experimental conditions on large transverse momenta and large invariant masses. The majority of the electron-positron pairs is produced with a invariant mass of the order of 1-10 $m_{e}$ with rather small $p_{t}$. Also the photon-photon mechanism is strongly peaked at very forward angles and therefore $\gamma \gamma$ pairs will show up prominently under such kinematical conditions

We extended our calculations also to RHIC energies. In this energy regime, $\gamma \gamma$ pairs are produced with invariant masses up to several $\mathrm{GeV}$. Therefore the $\gamma \gamma$ mechanism can be potentially dangerous in this case. Again, our calculations show that this is only a small effect in the central region, where we compare our calculations with the predictions given in [14]. In any case, with the present calculations the contributions due to the $\gamma \gamma$ mechanism are well under control. They are reassuringly small in the central region, where one is looking for the effects of hot and dense matter.
[1] G. Baur, K. Hencken, and D. Trautmann, J. Phys. G 24, 1657 (1998).

[2] K. Hencken, P. Stagnoli, D. Trautmann, and G. Baur, in Proceedings of the Photon'99 Conference, Freiburg, 1999, edited by S. Söldner-Rembold [Nucl. Phys. B (to be published)].

[3] G. Agakichiev et al., Phys. Rev. Lett. 75, 1272 (1995).

[4] B. Lenkeit for the CERES Collaboration, Proceedings of the International Nuclear Physics Conference, Paris, 1998.

[5] K. Hencken, D. Trautmann, and G. Baur, Phys. Rev. A 49, 1584 (1994).

[6] K. Hencken, D. Trautmann, and G. Baur, Phys. Rev. A 51, 1874 (1995).

[7] G. Baur, and N. Baron, Z. Phys. C 60, 95 (1993).

[8] T. Lippert, J. Thiel, N. Grün, and W. Scheid, Int. J. Phys. 6, 5249 (1991).

[9] G.P. Lepage, J. Comput. Phys. 27, 192 (1978).
[10] G.P. Lepage, Cornell Laboratory for Nuclear Sciences Report No. CLNS-80/447, 1980.

[11] G. Baur and L.G. Ferreira Filho, Phys. Lett. B 254, 30 (1991).

[12] M.E. Peskin and D.V. Schroeder, An Introduction to Quantum Field Theory (Addison-Wesley, Redwood City, CA, 1995).

[13] R. Hayano et al., in 4th Workshop on Experiments and Detectors for a Relativistic Heavy Ion Collider, 1990, edited by M. Fatyga and B. Moskowitz (1990), Vol. BNL 522262.

[14] R. Rapp, in Proceedings of Quark Matter 99, Turino (Italy), 1999 [Nucl. Phys. A (to be published)]; see also http://www.qm99.to.infn.it/rhic_pred/rapp/rapp.html

[15] V.B. Berestetskii, E.M. Lifshitz, and L.P. Pitaevskii, in Quantum Electrodynamics, No. IV in Course of Theoretical Physics, edited by L.D. Landau and E.M. Lifshitz (Pergamon, Oxford, 1982). 